\title{
Inherent photoluminescence Stokes shift in GaAs
}

\author{
Bruno Ullrich, ${ }^{1,2,5, *}$ Akhilesh K. Singh, ${ }^{1}$ Puspendu Barik, ${ }^{1}$ Haowen $\mathrm{Xi}^{3}{ }^{3}$ and Mithun Bhowmick ${ }^{4}$ \\ ${ }^{1}$ Instituto de Ciencias Físicas, Universidad Nacional Autónoma de México, Cuernavaca, Morelos 62210, Mexico \\ ${ }^{2}$ Ullrich Photonics LLC, Wayne, Ohio 43466, USA \\ ${ }^{3}$ Department of Physics and Astronomy, Bowling Green State University, Bowling Green, Ohio 43403-0209, USA \\ ${ }^{4}$ School of Science and Technology, Nazarbayev University, Astana 010000, Kazakhstan \\ ${ }^{5}$ e-mail: bruno@fis.unam.mx \\ ${ }^{*}$ Corresponding author: bruno.ullrich@yahoo.com
}

Received February 26, 2015; revised April 29, 2015; accepted May 12, 2015; posted May 12, 2015 (Doc. ID 235243); published May 28, 2015

\begin{abstract}
The intrinsic photoluminescence Stokes shift, i.e., the energy difference between optical band gap and emission peak, of $350 \mu \mathrm{m}$ thick semi-insulating GaAs wafers is found to be $4 \mathrm{meV}$ at room temperature. The result is based on the determination of the optical bulk band gap from the transmission trend via modified Urbach rule whose result is confirmed with the transmission derivative method. The findings reveal the detailed balance of the optically evoked transitions and disclose the intrinsic link between Stokes shift and the Urbach tail slope parameter. (c) 2015 Optical Society of America

OCIS codes: (300.6360) Spectroscopy, laser; (300.2530) Fluorescence, laser-induced; (300.6470) Spectroscopy, semiconductors; (300.6450) Spectroscopy, Raman.

http://dx.doi.org/10.1364/OL.40.002580
\end{abstract}

When a solid undergoes an electronic transition, it typically changes the vibrational state. This leads to the concept of vibronic (i.e., vibrational-electronic) transitions [1]. Electronic transitions can be provoked by various energy providing mechanisms, while here-in we focus on the optical excitation of the III-V compound semiconductor GaAs. The interaction of the electronic states of the atoms in a solid with the vibrational lattice modes, usually called electron-phonon coupling causes as a consequence of the vibrational energy consumption, a redshift of the photoluminescence (PL) peak with respect to the fundamental transition. In general, the degradation of the optical energy is called Stokes shift [2]. Although the phenomenon has been known since the nineteenth century for atomic transitions and multiple decades for semiconductors (particularly for GaAs) [1-ㄱ] , we are not aware of attempts to pinpoint the intrinsic chromaticity defining PL Stokes shift of GaAs. In the current Letter, augmenting our previous works on p-type GaAs [4], CdS [5], and $\mathrm{ZnO}$ [6], we identify the inherent GaAs Stokes shift of semi-insulating (SI) GaAs. Additionally, the work emphasizes the association of the Urbach tail and Stokes shift in predominately covalently bonded semiconductors.

The here-in used undoped $\langle 100\rangle$ oriented $350 \mu \mathrm{m}$ thick SI-GaAs wafer (lot number 8290-1) was provided by Semiconductor Wafers Inc. (SWI). The material's quality was checked by Raman spectra excited at room temperature (RT) with a He-Cd laser at $442 \mathrm{~nm}$. The results of the longitudinal and transversal mode measurements are shown in Fig. 1, which confirms the GaAs wafer's single crystal zinc blende structure by the observation of pure transverse optical (TO) and longitudinal optical (LO) peaks located at $268 \mathrm{~cm}^{-1}$ and $292 \mathrm{~cm}^{-1}$ [7]. The PL was excited at $266 \mathrm{~nm}$ with a vertically polarized pulsed (11 ns, $22 \mathrm{kHz}$ ) solid-state laser from Laserglow Inc., whereas the emission was recorded with Ocean Optics (USB 2000) and StellarNet (C-SR-200) fiber spectrometers. The transmittance (TR) of the wafers was detected via lock-in techniques by exciting the sample with a
$100 \mathrm{~W}$ tungsten-halogen lamp in conjunction with the Newport Cornerstone $2601 / 4 \mathrm{~m}$ monochromator. All experiments were carried out at RT.

The determination of the intrinsic Stokes energy displacement $\left(\Delta_{\text {Stokes }}\right)$ between the energy locations of the optical band gap $\left(E_{g, o}\right)$ and the PL peak $\left(E_{\mathrm{PL}}\right)$ requires measurements at RT to favor band-to-band recombination upon the formation of excitons and strong TR signals to be able to precisely point out the value of $E_{g, o}[\underline{5}$ ]. The latter condition is the major obstacle because, as in the current work, top-quality crystalline materials possess thicknesses exceeding by far the single digit $\mu \mathrm{m}$ range. To elude that specific difficulty, we present two ways to determine $E_{g, o}$ of bulk semiconductors, i.e., by using the modified Urbach rule [6] or the derivative $d T R / d h \nu$, where $h \nu$ is the impinging photon energy []. So far, these methods have been applied to $\mathrm{ZnO}$ and $\mathrm{GaSb}$, respectively. Here, we demonstrate that both procedures deliver matching $E_{g, o}$ values for bulk GaAs.

The broken line in Fig. 2 represents the fit of the TR data (represented by the circular symbols) carried out with Beer's law [2]

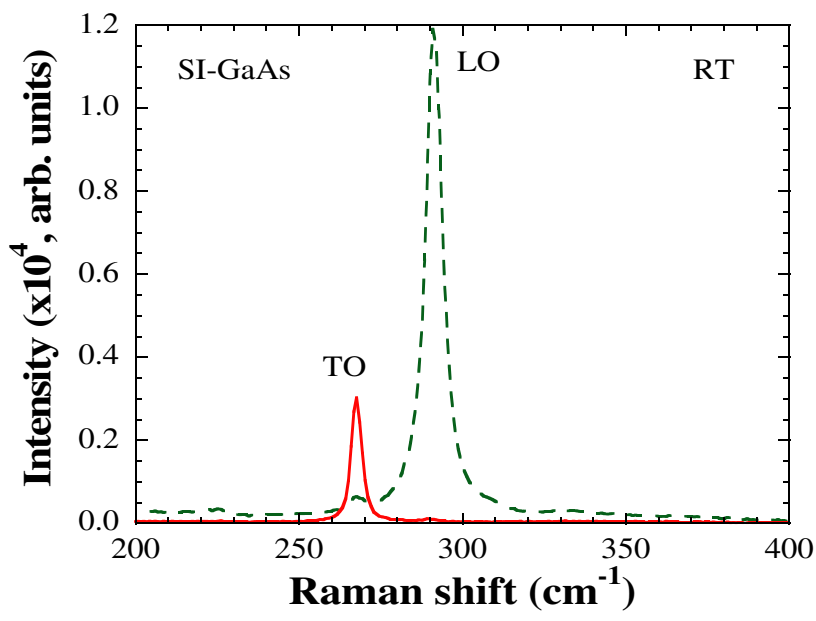

Fig. 1. Raman spectra of the investigated SI-GaAs wafer. 


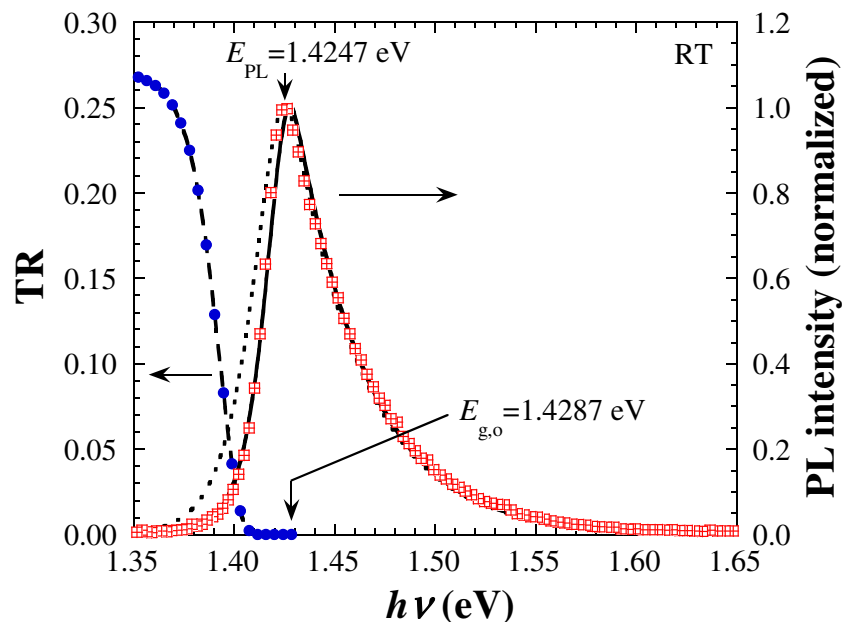

Fig. 2. TR and PL spectra of the SI-GaAs wafer. The symbols and lines represent the measured data and fits, which are described in detail in the text.

$$
\operatorname{Tr}(h \nu)=C \times e^{-\alpha(h \nu) d}
$$

where $C$ is a scaling constant taking into account scattering losses and the reflected light, $d$ is the thickness of the wafer, and $\alpha$ is the absorption coefficient expressed by the modified Urbach rule [9],

$$
\alpha(h \nu)=A\left[\frac{k T}{2 \sigma_{a}}\right]^{1 / 2} e^{\frac{\sigma_{a}\left(h \nu-E_{\mathrm{cr}}\right)}{k T}}
$$

where $A$ is a constant on the order of $\sim 10^{4} \mathrm{~cm}^{-1}(\mathrm{eV})^{-1 / 2}$, $k T=0.025 \mathrm{eV}$ is the ambient thermal lattice energy, $\sigma_{a}$ is a dimensionless parameter defining the slope of the absorption edge, and $E_{\mathrm{cr}}$ is the energy where the absorption crossover between density of states and the Urbach tail occurs. It has been pointed out in Ref. [6] that $E_{\text {cr }}$ is identical with $E_{g, o}$. The well matching TR fit $\left(\chi^{2}=0.999\right)$, performed with Eqs. (1) and (2), gives $E_{g, o}=1.4287 \pm$ $0.0080 \mathrm{eV}, \quad A=2.1 \times 10^{4} \pm 2 \times 10^{3} \mathrm{~cm}^{-1}(\mathrm{eV})^{-1 / 2}, \quad$ and $\sigma_{a}=2.73 \pm 0.10$. Figure 3 shows the TR in a logarithmic plot revealing how the fit comes about: the deviation minimization between the TR trend in the transmissive region and Eq. (1) above $\sim 1.41 \mathrm{eV}$, which marks the point of transparency of the wafer, requires the movement of $E_{g . o}$ into the highly opaque range, precisely pinpointing the crossover energy between the Urbach tail and the density of states [6].

To check the $E_{g, o}$ value found by the fit in Fig. 2, we applied the method outlined in Ref. [8].The symbols in Fig. 4 show the numerical derivative $d T R / d h \nu$ of the measured TR spectrum in Fig. 2 and its Gaussian fit $\left(\chi^{2}=0.971\right)$. The latter reveals the values of the center energy of the Gaussian distribution and width parameter to be $c=$ $1.3930 \pm 0.0005 \mathrm{eV}$ and $w=0.0089 \pm 0.0005 \mathrm{eV}$, resulting in $E_{g, o}=c+4 w=1.4286 \pm 0.0025 \mathrm{eV}$ [ [8]. The value coincides well with the above result and agrees with the RT GaAs band gap energy of $1.43 \mathrm{eV}$ given in the literature $[2,4,10]$.

It is imperative to check the cohesiveness of the TR and PL data in Fig. 2 to ensure the intrinsic nature of $\Delta_{\text {Stokes }}$. Ideally, the experimental PL result, which is

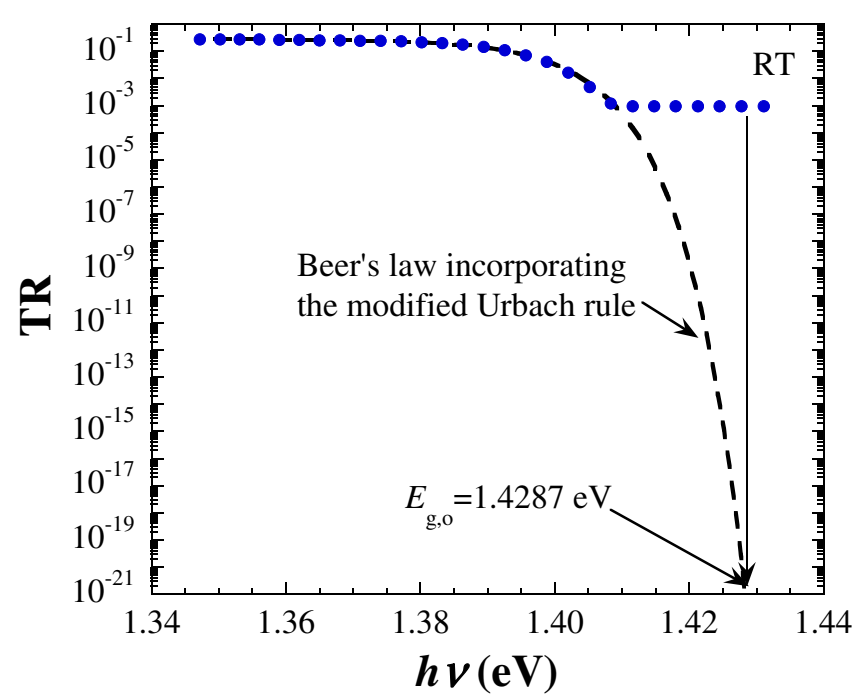

Fig. 3. Logarithmic TR plot. The symbols are identical with those in Fig. 2. The broken line shows the entire fit done with Eq. (1). The optimized TR slope fit for energies above $\sim 1.41 \mathrm{eV}$ is achieved when $E_{g, o}$ is located at $1.4287 \mathrm{eV}$, where the wafer is completely opaque.

represented by the square symbols in Fig. 2 pointing to $E_{\mathrm{PL}}=1.4247 \pm 0.0011 \mathrm{eV}$, ought to be excited with a non-lasing moderate optical stimulus to avoid distortion of the inherent recombination channels [5]. However, in contrast to $\mathrm{ZnO}$ crystals and doped GaAs wafers, we were not able to excite RT PL of SI-GaAs using Xenon lamp sources. Therefore, we altered the PL exciting average laser intensities by more than one order of magnitude $\left(10-400 \mathrm{~mW} / \mathrm{cm}^{2}\right)$ to check whether $E_{\mathrm{PL}}$ changes. Within this intensity range we did not observe a shift of $E_{\mathrm{PL}}$ and, while we stress that both employed fiber monochromators recorded the same $E_{\mathrm{PL}}$ position within the error bars, the ongoing discussion below demonstrates that the absorption basically defines the emission. In other words, the detailed balance principle holds

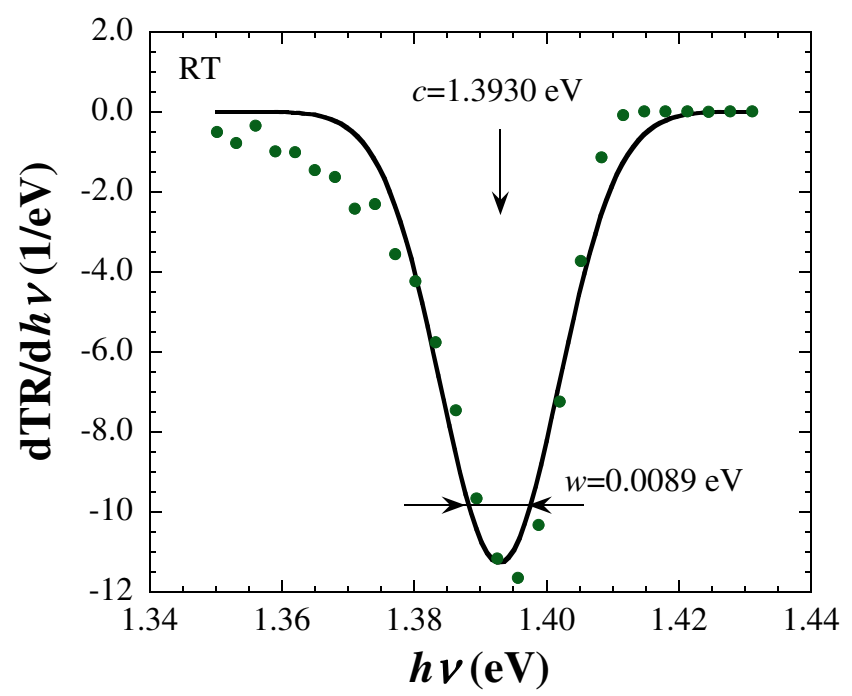

Fig. 4. The circular symbols and solid line show the numerical derivative $d T R / d h \nu$ of the measured TR spectrum displayed in Fig. $\underline{2}$ and the Gaussian fit, respectively. 
[2,11], emphasizing once more the intrinsic link between the results in Fig. 2 .

The remarkable outcome of the TR fit in Fig. 2 is that it provides the key parameter set required for the expression $[\underline{6}, \underline{9}]$

$$
\alpha(h \nu)=A\left[h \nu-E_{g, o}+\frac{k T}{2 \sigma_{a}}\right]^{1 / 2},
$$

which defines $\alpha(h \nu)$ beyond the Urbach tail into the density of states region for $h \nu>E_{g, o}$. Having now the $\alpha(h \nu)$ dispersion available, by employing the extended Roosbroeck-Shockley relation (RSR) $[\underline{4}, \underline{6}, \underline{12}]$, we fit the PL spectrum intensity with

$$
I_{\mathrm{PL}}(h \nu) \propto[h \nu]^{2} \times \frac{1-e^{-\alpha(h \nu) d_{\mathrm{eff}}}}{e^{\frac{h \nu}{k T_{c}}}-1},
$$

where $d_{\text {eff }}$ is the reduced effective sample thickness and $k T_{c}$ is the actual carrier energy which, in general, exceeds $k T$ [13]. With the parameters $d_{\text {eff }}=2.4 \times$ $10^{-3} \pm 2 \times 10^{-4} \frac{\mathrm{cm}}{\mathrm{cm}}$, which correspond to the electron diffusion length in GaAs [14], and $k T_{c}=0.034 \pm 0.002 \mathrm{eV}$ [15], we find the fit $\left(\chi^{2}=0.999\right)$ visualized with the solid line. However, in contrast to the PL fit of $\mathrm{ZnO}$ [6], to best theoretically match the PL spectrum, we had to modify the $\sigma_{a}$ value to $\sigma_{\mathrm{PL}}=3.50 \pm 0.10$ (which is the only parameter alteration with respect to the TR fit). For comparison, the dotted line shows the calculated PL spectrum with $\sigma_{a}=2.73$. Hence, as found with p-type GaAs as well [4], the PL fit delivers for the lower energies (below $\sim 1.4 \mathrm{eV}$ ) the material's recombination edge rather than its absorption edge.

Using $E_{g, o}=1.4287 \mathrm{eV}$, we find $\Delta_{\text {Stokes }}=E_{g, o}-E_{\mathrm{PL}}=$ $4.0 \mathrm{meV}$ which, by considering the above pointed out errors of $\pm 8.0 \mathrm{meV}$ and $\pm 1.1 \mathrm{meV}$, respectively, may lead to the anti-Stokes value of $\Delta_{\text {Stokes }}=-5.1 \mathrm{meV}\left(E_{\mathrm{PL}}>\right.$ $\left.E_{g, o}\right)$ and to the maximum possible Stokes energy displacement of $\Delta_{\text {Stokes }}=13.1 \mathrm{meV}$. Within this scatter, the theoretical value of $\Delta_{\text {Stokes }}$ is expected. The latter is expressed with the formula below incorporating Fan's classical relation for the semiconductor's selfenergy $[\underline{5}, \underline{16}]$ :

$$
\Delta_{\text {Stokes }}=\left(\frac{1}{\varepsilon_{\infty}}-\frac{1}{\varepsilon_{0}}\right)\left[\sqrt{\left(\frac{m_{e}}{m_{0}}\right)}+\sqrt{\left(\frac{m_{h}}{m_{0}}\right)}\right] \sqrt{\left|E_{\mathrm{Ryd}}\right| h \nu_{\mathrm{LO}}},
$$

where $\varepsilon_{\infty}(11.6)$ is the high frequency and $\varepsilon_{0}$ (12.5) the static dielectric constant [17], $m_{0}$ is the free electron mass, $m_{e}$ and $m_{h}$ are the effective electron (0.067) and hole (0.5) masses [10], $E_{\text {Ryd }}$ is the Rydberg energy $(-13.6 \mathrm{eV})$, and $h \nu_{\mathrm{LO}}$ is the LO phonon energy (292 $\mathrm{cm}^{-1}=36.2 \mathrm{meV}$ according to Fig. 1), resulting in $\Delta_{\text {Stokes }}=4.2 \mathrm{meV}$, matching the above findings. The result reveals the influence of the crystal bonding, i.e., ionic versus covalent, on the Stokes shift value [1]. While $\mathrm{CdS}$ and $\mathrm{ZnO}$ exhibit $\Delta_{\text {Stokes }}>h \nu_{\mathrm{LO}}[\underline{5}, \underline{6}]$, for GaAs, $\Delta_{\text {Stokes }} \ll$ $h \nu_{\mathrm{LO}}$, because of the proximity of the dielectric constants. The extremes with $\varepsilon_{\infty}=\varepsilon_{0}$ are the element semiconductors $\mathrm{Ge}$ and $\mathrm{Si}$ for which $\mathrm{Eq}$. (5) gives
$\Delta_{\text {Stokes }}=0 \mathrm{eV}$. We did not find specific Stokes shift studies in the literature for these solids, but for hydrogenated amorphous Si no Stokes shift was observed [18]. Consequently, the blend of a vibronic system with the solid's polarity, which is a typical feature of compound semiconductors, is the origin of a perceptible Stokes shift. We further note that $\Delta_{\text {Stokes }}$ can be influenced by doping. For heavily $\mathrm{Si}$ and $\mathrm{Zn}$ doped GaAs with dopant concentrations of $\sim 10^{18} \mathrm{~cm}^{-3}$, we found that n-type doping slightly increases the Stokes shift $\left(\Delta_{\text {Stokes }}=4.7 \mathrm{meV}\right)$, while p-type doping bleaches the Stokes energy displacement $\left(\Delta_{\text {Stokes }} \sim 0 \mathrm{eV}\right)$. The subject of the $\Delta_{\text {Stokes }}$ dependence on doping levels merits further investigation.

Based on the findings here, we are able to render the relation between the Urbach tail and the Stokes shift. Knowing that the Stokes shift can be measured in terms of the Gaussian dispersion $w^{2}$ of the absorption edge [19], whose number is determined by the fit in Fig. $\underline{4}$, we establish that $\Delta_{\text {Stokes }}=k T /\left(2 \sigma_{a}\right) \sim w^{2} / k T$ for covalently bonded semiconductors. Indeed, reflecting good harmony with the result of Eq. (5), the expressions generate the $\Delta_{\text {Stokes }}$ numbers of 4.6 and $3.2 \mathrm{meV}$, respectively.

In conclusion, the RT inherent PL Stokes shift of highquality industrially produced undoped SI-GaAs wafers is pointed out to be $4 \mathrm{meV}$, i.e., almost one order of magnitude below the optical phonon energies. Its determination was enabled by employing the modified Urbach tail formula and the Gaussian function method to locate precisely the bulk value of $E_{g, o}$. The presented result, which is in agreement with Fan's classical expression, demonstrates that the GaAs PL is ruled by the RSR and further reveals the validity of $\Delta_{\text {Stokes }}=k T /\left(2 \sigma_{a}\right)$ for covalent semiconductors.

The work was partially supported by the DGAPAUNAM PAPIIT project TB100213-RR170213, PI Bruno Ullrich. AKS and PB acknowledge the DGAPA-UNAM program of post-doctoral fellowships. The authors are indebted to Dr. Jens W. Tomm at the Max-Born-Institut (MBI) in Berlin for the measurements of the Raman spectra.

\section{References and Notes}

1. M. Fox, Optical Properties of Solids, 2nd ed. (Oxford University, 2010).

2. J. I. Pankove, Optical Processes in Semiconductors (Dover, 1971).

3. E. W. Williams and A. M. White, Solid State Commun. 9, 279 (1971).

4. B. Ullrich, S. R. Munshi, and G. J. Brown, Semicond. Sci. Technol. 22, 1174 (2007).

5. B. Ullrich, D. Ariza-Flores, and M. Bhowmick, Thin Solid Films 558, 24 (2014).

6. B. Ullrich, A. K. Singh, M. Bhowmick, P. Barik, D. ArizaFlores, H. Xi, and J. W. Tomm, AIP Adv. 4, 123001 (2014).

7. T. Miyoshi, S. Iwai, Y. Iimura, Y. Aoyagi, and S. Namba, Jpn. J. Appl. Phys. 29, 1435 (1990).

8. B. Ullrich, G. J. Brown, and H. Xi, Semicond. Sci. Technol. 27, 105016 (2012).

9. B. Ullrich and C. Bouchenaki, Jpn. J. Appl. Phys. 30, L1285 (1991).

10. B. G. Streetman and S. K. Banerjee, Solid State Electronic Devices (Prentice Hall, 2006).

11. W. van Roosbroeck and W. Shockley, Phys. Rev. 94, 1558 (1954). 
12. B. Ullrich, S. Yano, R. Schroeder, and H. Sakai, J. Appl. Phys. 93, 1914 (2003).

13. B. Ullrich, D. M. Bagnall, H. Sakai, and Y. Segawa, Solid State Commun. 109, 757 (1999).

14. L. W. Aukerman, M. F. Millea, and M. McColl, J. Appl. Phys. 38, 685 (1967).

15. Fitting the high-energy PL tail in the energy range of $1.45-1.60 \mathrm{eV}$ in Fig. 2 with $I_{\mathrm{PL}}(h \nu) \propto \exp \left(-h \nu / k T_{c}\right)$ gives $k T_{c}=0.0376 \pm 0.000 \overline{1} \mathrm{eV}$, satisfactorily matching the $k T_{c}$ fit result found with the RSR.
16. H. Y. Fan, Photon-Electron Interaction: Crystals Without Fields (Springer, 1967), Vol. 5/25/2, pp. 157-233.

17. In Eq. (5), the parameter with the most widely spread values is $\varepsilon_{0}$, S. Adachi Ed., Properties of Aluminum Gallium Arsenide (INSPEC, 1993), which leads to an error of at least $50 \%$, i.e., $\Delta_{\text {Stokes }}=$ $4.2 \pm 2.1 \mathrm{meV}$.

18. D. Dunstan and F. Boulitrop, J. Phys. Colloq. 42, C4-331 (1981).

19. P. G. Eliseev, J. Appl. Phys. 93, 5404 (2003). 